\title{
Attentional selection during preparation of prehension movements
}

\author{
Andreas Schiegg, Heiner Deubel, and Werner X. Schneider \\ Department Psychologie, Allgemeine und Experimentelle Psychologie, \\ Ludwig-Maximilians-Universität, München, Germany
}

\begin{abstract}
In two experiments coupling between dorsal attentional selection for action and ventral attentional selection for perception during preparation of prehension movements was examined. In a dual-task paradigm subjects had to grasp an Xshaped object with either the left or the right hand's thumb and index finger. Simultaneously a discrimination task was used to measure visual attention prior to the execution of the prehension movements: Mask items transiently changed into distractors or discrimination targets. There was exactly one discrimination target per trial, which appeared at one of the four branch ends of the object. In Experiment 1 target position varied randomly while in Experiment 2 it was constant and known to subjects in each block of trials. In both experiments discrimination performance was significantly better for discrimination target positions at to-begrasped branch ends than for not-to-be-grasped branch ends. We conclude that during preparation of prehension movements visual attention is largely confined to those parts of an object that will be grasped.
\end{abstract}

Visual information processing in the primate brain can be divided up into two streams. Goodale and Milner (1992) suggested that the dorsal stream is involved in computation of information for spatial motor actions while computations for visual perception and identification take place in the ventral stream. There have been numerous studies focusing on the "identification" -aspect of visual processing. By selecting a certain object or region of the visual field ("selection for perception"') visual attention is assumed to facilitate detection (e.g., Posner, 1980), finding targets in an environment of distractors (see, e.g., Wolfe, 1994, for an overview), integration of features from different visual modules into object files (Treisman \& Gelade, 1980), to allow recognition of objects (e.g.,

Please address all correspondence to A. Schiegg, Department Psychologie, Allgemeine und Experimentelle Psychologie, Ludwig-Maximilians-Universität, Leopoldstr. 13, 80802 München, Germany. E-mail: schiegg@psy.uni-muenchen.de

AS would like to thank Paul Parkinson for his comments on an earlier draft of the manuscript. This research was supported by the Deutsche Forschungsgemeinschaft, SFB 462 ("'Sensomotorik").

(C) 2003 Psychology Press Ltd

http://www.tandf.co.uk/journals/pp/13506285.html

DOI:10.1080/13506280244000140 
LaBerge \& Brown, 1989), and to regulate entry into visual short-term memory (Bundesen, 1990; Duncan \& Humphreys, 1989).

As for computation in the dorsal stream, programming a motor activity such as reaching or grasping an object also requires some kind of selection of this object among others being irrelevant to the particular task. This was first proposed by Allport (1987) and Neumann (1987) and termed "selection for action" (Allport, 1987). A number of models suggest that visual attention is involved in this selection process, too. According to the premotor hypothesis, control of "spatial attention" originates in the dorsal spatial motor areas, which results in a shift of visual attention towards the target during programming of a movement. Originally proposed for eye movements (Rizzolati, Riggio, Dascola, \& Umiltà, 1987) this idea was later generalized to all goaldirected spatially coded movements (Rizzolati, Riggio, \& Sheliga, 1994). The integrated competition hypothesis by Duncan (1996) considers attention to be an emerging state in which visual representations of one object win the competition against representations of other objects. Biasing the competition towards one object is assumed to be controlled by the current task instruction and to originate in brain areas where the task-relevant attributes are computed. Therefore an object-specific coupling between ventral and dorsal stream is predicted. Like the integrated competition hypothesis, the Visual-AttentionModel (VAM) of Schneider (1995) suggests a common selection mechanism for both processing streams. In this model, visual attention is thought to select one low-level visual object at a time, leading to prioritized perceptual processing in the ventral stream. Simultaneously, possible spatial motor actions (saccading, pointing, reaching, grasping) towards this object are programmed in the dorsal stream. According to VAM, during the programming of a motor activity visual attention should always be largely confined to the object relevant for the motor task being programmed. This has already been shown for saccades (Deubel \& Schneider, 1996; Hoffmann \& Subramaniam, 1995; Kowler, Anderson, Dosher, \& Blaser, 1995; Schneider \& Deubel, 2002) and manual reaching movements (Deubel, Schneider, \& Paprotta, 1996, 1998). In the latter, subjects had to execute a pointing movement to a peripheral target object, indicated out of a number of potential target stimuli by a central visual cue. Discrimination performance measured during preparation of the movement was by far best at the position of the movement target, and poor for other target positions.

There are also a number of studies dealing with attentional selection processes for reach-to-grasp movements. Common to these studies is the presence of distractor objects whose interference on kinematic parameters of reach-tograsp movements to the target object is examined. It has been proposed (Jeannerod, 1981) that grasping movements consist of two components, controlled by separate visuomotor channels: The transport component can most simply be described as "bringing the hand to the target object's position", whereas the 
manipulation component deals with actually grasping the object and therefore requires computation of parameters such as grasping aperture.

No interference effects on both components were found when the distractor was of no relevance to the reach-to-grasp movement, i.e., subjects knew position and identity of the object to be grasped before executing the task and therefore there was no need to attend to the distractor (Jackson, Jackson, \& Rosicky, 1995). Tipper, Howard, and Jackson (1997) reported interference effects of distractors on movement time and path of reach-to-grasp-movements using a task where subjects had to grasp a target object as fast and accurately as possible in the presence of distractor objects. However, these effects vanished when subjects knew in advance what the target was. In another study (Castiello, 1996), subjects had to grasp an object, while counting the number of times a distractor object was illuminated by a spotlight. Results showed that the type of distractor influenced the amplitude of the peak grip aperture, that is the manipulation component of the movement. When the distractor was smaller/bigger than the target, the peak aperture was also smaller/bigger than in trials without a distractor. Bonfiglioli and Castiello (1998) had subjects grasp an object while at the same time they had to attend to a moving distractor of the same size (because under certain conditions it could become the target object) either overtly or covertly. Interference effects on the transport component were found for the case of covert attention. In this experiment no effects were found for the manipulation component of the movement, supposedly because target and distractor involved programming the same parameters for their manipulation components. This view is supported by results of Kritikos, Bennett, Dunai, and Castiello (2000) who varied distractor size and found that only distractors whose size was different from the target's size caused interference effects.

Taken together these results suggest that in cases where visual attention is required for tracking or monitoring the distractor at the same time information seems to be passed on to the visuomotor channels interfering with parameters computed for the target object, yielding a nonindependency of selection for action and visual attention in perception for parameters relevant to both components - transport and manipulation — of prehension movements.

The purpose of the present study is to extend the above findings on coupling and dependency of selection for action and selection for perception to selection processes "within" one single object in the course of the computation of kinematic parameters prior to prehension movements. All of the above studies deal with attentional selection processes using tasks that require the selection of one object, the movement-relevant target, out of others, the movement-irrelevant distractors. However, grasping of one single object without the presence of distractor objects already requires a distinction between "relevant" and "irrelevant'". Assuming an object will be grasped using thumb and index finger, the locations which these two fingers are intended to land on are more relevant to the programming of the prehension movement than other parts of the same 
object. In addition to the required distinction between relevant and irrelevant locations, there are per se two relevant parts of the target that have to be taken into account in order to program the movement correctly, which is not the case for simple pointing-only tasks. In two experiments we will show that there are selection processes acting "within" an object facilitating movement-relevant parts (that is landing positions of the fingers the subjects grasp with) and, moreover, that there is an obligatory coupling between attentional selection for perception (visual attention) and attentional selection for action causing visual attention to be confined to the movement-relevant parts of the object during preparation of prehension movements.

\section{EXPERIMENT 1}

\section{Method}

Subjects. Six right-handed subjects, aged 20 to 33 years, participated in Experiment 1. All had normal vision and were experienced in various experiments involving manual pointing tasks and the same discrimination paradigm as used in this study. However, they were naïve with respect to the aim of this study and had not participated in experiments involving prehension tasks before.

Experimental set-up. The experimental set-up is sketched in Figure 1. The experiments were controlled by an IBM-compatible personal computer using custom software for stimulus presentation and online data recording. Stimuli were presented on a 21 -inch colour monitor (CONRAC 7550C21) with $40 \times$ $30 \mathrm{~cm}$ active screen size, receiving its input from a TIGA graphics board (KONTRAST 8000 ), that provided a resolution of $1024 \times 768$ pixels at a frame rate of $100 \mathrm{~Hz}$. Subjects had to grasp a wooden " $X$ "'-shaped object (see Figure 1b) which was mounted in the centre of a slightly inclined plane ("grasping plane") in front of them. The display consisted of the outline of the object and the visual stimuli used for measuring discrimination performance, which appeared with a luminance of $12 \mathrm{~cd} / \mathrm{m}^{2}$ on a gray background having a mean luminance of $1 \mathrm{~cd} / \mathrm{m}^{2}$. Prehension movements were executed on the grasping plane underneath a one-way mirror. Visual information was presented via the mirror which was adjusted such that stimuli were projected onto the grasping plane (see Figure 1a). By using this set-up it was possible to present subjects with discrimination targets and the outline of the object without having the disadvantage of the hands obstructing the subjects' view when moving across parts of the stimuli. Actual viewing distance was $60 \mathrm{~cm}$. Eye fixation was controlled by an SMI-Eyelink infrared eye monitoring system providing eyeposition data with a frequency of $250 \mathrm{~Hz}$ and a maximum overall delay of $10 \mathrm{~ms}$. Head movements were restricted by a chin-rest. An automatic trial by trial drift correction together with the built-in head-position compensation mechanism did 
(a)

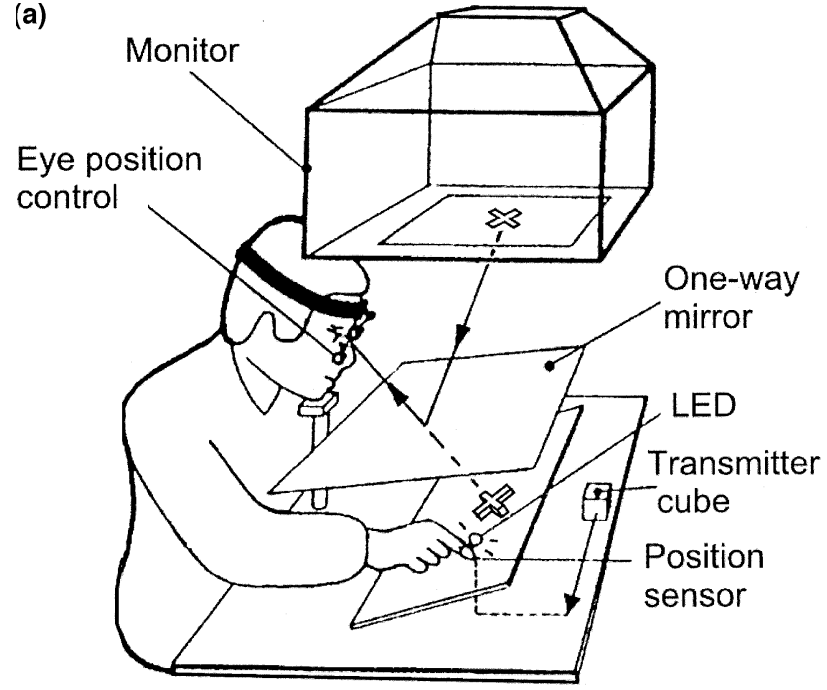

(b)

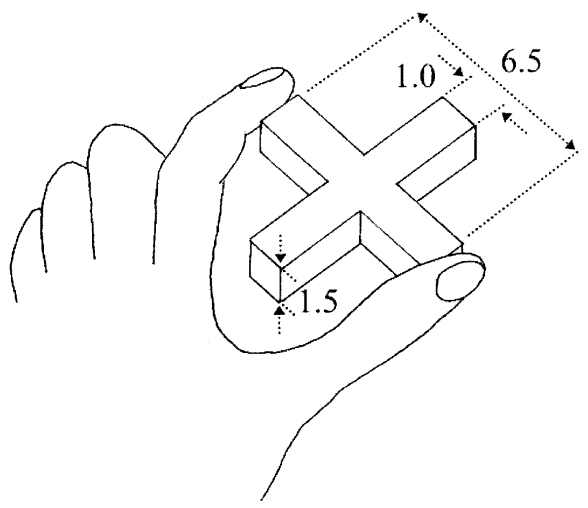

Figure 1. (a) Experimental apparatus. (b) Dimensions (in centimetres) of the object-to-be-grasped. The two bars intersect at an angle of $90^{\circ}$. The drawing shows the object grasped by the left hand.

not require any further restrictions. Prehension movements were recorded with a Polhemus Fastrak electro-magnetic position and orientation measuring system. The system provides the spatial position of a small position sensor relative to a transmitter cube. One sensor was mounted on the fingertip of the subjects' left and right hand index finger while the transmitter was fixed at $60 \mathrm{~cm}$ in front of the subjects. By spatial transformation the sensor coordinates were transformed into the reference frame of the projected monitor image. The device allows for a 
maximum translation range of $3 \mathrm{~m}$, with an accuracy of $1 \mathrm{~mm}$ RMS. The frequency response is $60 \mathrm{~Hz}$ using two sensors at a time; without further filtering the phase lag response is only $4 \mathrm{~ms}$. The position sensor behaved linearly within $30 \mathrm{~cm}$ around the centre position. The overall accuracy was better than $2 \mathrm{~mm}$. Attached to each sensor was a small red LED. These LEDs allowed to provide controlled visual feedback about the spatial position of the fingertip used by subjects for hand positioning at the beginning of each trial.

Spatial layout of display. The basic layout is depicted in Figure 2(a). In the centre of the screen there was a fixation cross $\left(0.25 \times 0.25^{\circ}\right)$ surrounded by the outline of the " $\mathrm{X}$ "'-shaped object to be grasped which was $6.3^{\circ}$ of visual angle in diameter. Though we could not give subjects an impression of the object's depth by only displaying its outline, they had no difficulties grasping it. Sixteen mask items $\left(0.86 \times 0.57^{\circ}\right.$ in size $)$ were placed equally spaced at $3.35^{\circ}$ eccentricity on a virtual circle around the object's centre, with one item being at the end of each branch of the " $X$ ' and three between neighbouring branches.

There were three different types of mask items (see Figure $2 b$ ) one of which was randomly chosen for each trial. At the bottom of the display at a vertical distance of $11.5 \mathrm{~cm}$ from the centre of the object and $12 \mathrm{~cm}$ to the left and right of the vertical symmetry axis going through the centre of the display there were two rectangles $(1 \times 1 \mathrm{~cm})$ visible during the hand positioning period before each trial. They served as markers for the starting position of subjects' hands.

Discrimination task. A discrimination task was used to measure allocation of attention in the ventral stream during movement preparation. The mask items changed transiently into distractors (" 2 ", or " 5 ") and-one item per trial-into a discrimination target ("E" or " $\exists$ "). The time course will be described in detail in the Temporal Sequence of Stimuli section. At this point we would only like mention that our indicator for allocation of attention in the ventral stream was the accuracy with which the discrimination target could be identified. Discrimination performance was measured by computing the percentage of correct decisions of target identity. After each trial subjects had to press one of two keys, mounted $2 \mathrm{~cm}$ to the left and right of the vertical symmetry axis on a virtual line from one positioning marker to the other, to report the letter which they thought to have recognized (two alternative forced choice), pressing the right key for " $E$ "' and the left for " $\exists$ '. Keys were illuminated by LEDs at the end of each trial and therefore visible through the one-way mirror.

Prehension task. Subjects had to grasp the " $X$ ' -shaped object either with the left or the right hand's thumb and index finger, grasping the top left branch end with the index finger and the bottom right branch end with their thumb when using the left hand. For right-hand prehension movements the top right branch 
(a)
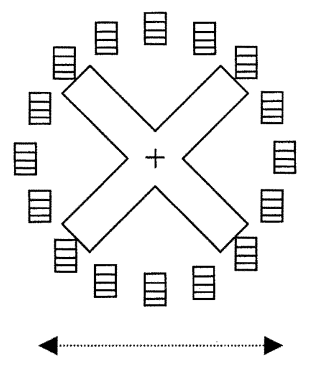

$7.7 \mathrm{~cm}=7.3^{\circ}$

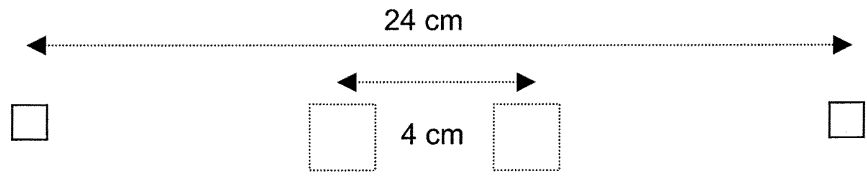

(b)

Mask items
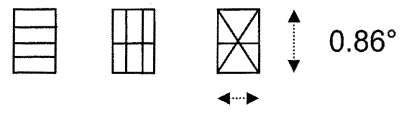

$0.57^{\circ}$

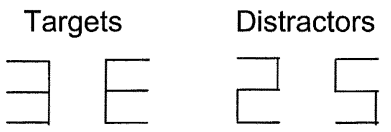

Figure 2. (a) Spatial layout of display. The dotted rectangles indicate the position of the two keys for reporting the recognized letter. (b) Mask items, targets, and distractors. Angles are visual angles for $60 \mathrm{~cm}$ viewing distance.

end had to be grasped with the index finger and the bottom left with the thumb respectively. Movement starting position together with object geometry and position (see below) was chosen such that the object could be grasped simply by moving the hand in a straight way and adjusting the correct grip aperture without bending the wrist.

The main purpose of the present study was an analysis of attentional selection of to-be-grasped parts of an object during preparation of movements. We had to make sure that processing the discrimination task did not interfere with movement preparation such that for example subjects would interrupt movement preparation when processing the discrimination target. Therefore, movement onset latency had to be independent of the discrimination target's position, i.e., grasping parts of the object where the discrimination target was 
presented versus grasping parts of the object where the discrimination target was not presented.

Moreover, it was important that subjects really grasped the indicated parts of the object. Otherwise one could not have assumed that, during movement preparation, the landing positions of the fingers would be selected if there were measurable selection processes. For this reason the second parameter we had to control was each finger's landing position. Pretesting had shown that for relatively simple prehension movements we had the subjects perform, one could very reliably assume that, if the index finger had reached its landing position correctly, the thumb had also done so. Furthermore, with respect to movement onset latency it did not matter whether thumb or index finger movement was evaluated. Therefore, to reduce data to be recorded and to avoid unnecessary movement restrictions and inconveniences for subjects we only recorded index finger positions, where choosing the index finger was due to technical reasons (we found the position sensors' cabling to be less disturbing for the subjects in this case).

In addition to movement onset latency and landing positions, movement duration was evaluated in order to be able to discard trials involving movements that lasted too long.

Temporal sequence of stimuli. The temporal sequence of stimuli is depicted in Figure 3. Subjects were told that the experiment consisted of two simultaneous tasks - grasping and discrimination-of which the former had priority. At the beginning of each trial there was a period of $1000 \mathrm{~ms}$ during which the hand positioning markers were displayed and the LEDs on both hands' index finger tips were turned on. Subjects were instructed to fixate the cross in the centre of the display and to place their hands on the grasping plane, with thumb and index finger slightly touching each other and the LEDs being within the marker-rectangles. This provided a well-defined equal starting position for both hands with respect to the object to be grasped. Position markers and LEDs were turned off and, after a delay of 600-1000 ms during which an automatic eye-position-drift correction was performed, an acoustic cue indicated the hand to grasp with. We used a beep of $100 \mathrm{~ms}$ duration, being of high frequency $(3800 \mathrm{~Hz})$ indicating the left hand to grasp with and low frequency $(500 \mathrm{~Hz})$ for the right hand. The beep also served as the movement go-signal. Subjects were instructed to grasp as fast and accurately as possible as soon as they heard the beep

$150 \mathrm{~ms}$ after the go-signal all mask items changed for $140 \mathrm{~ms}$ displaying " $\mathrm{E}$ " or " $\exists$ ", at exactly one (of four) branch ends as the discrimination target and " 2 ", or " 5 " at all other positions serving as distractors. The branch end where the discrimination target (DT) appeared was randomly chosen but balanced between trials. Subjects were told that the DT would appear randomly at one of the four branch ends in each trial. Targets and distractors were again replaced by mask 


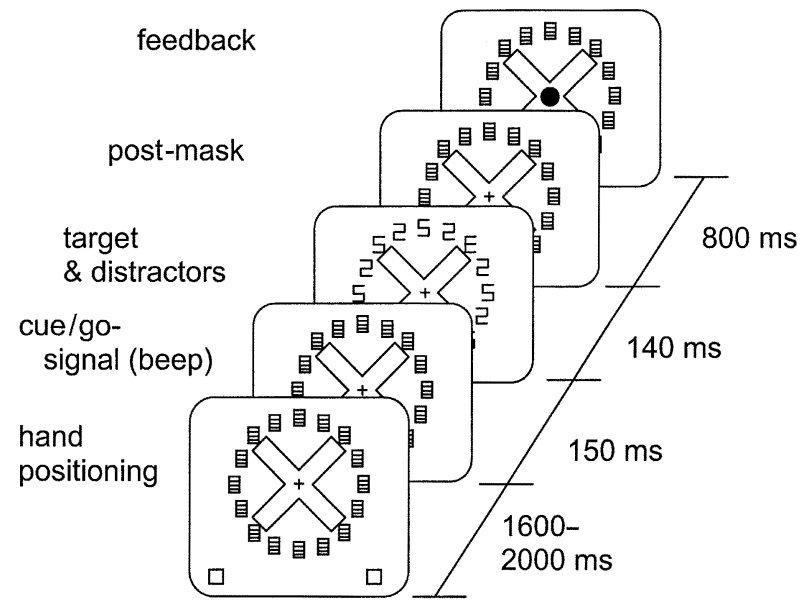

Figure 3. Temporal sequence of stimuli. The outer rectangles served as markers for the start positions of the hands. They were turned off after $1000 \mathrm{~ms}$, which is not shown in an extra frame.

items after the target presentation interval. After another $800 \mathrm{msec}$ which were used to record the remainder of the actual hand movement, feedback on onset latency of the prehension movement was given by a coloured dot in the centre of the screen, red meaning "too slow" (latency $>500 \mathrm{~ms}$ ), yellow "quite OK, but could be faster" (latency between 350 and $500 \mathrm{~ms}$ ) and green "OK" (latency $<350 \mathrm{~ms}$ ). As decribed above, at the end of the trial, subjects had to report the DT they thought to have seen by pressing one of two keys on a keypad. The keypress also started the next trial.

Data analysis and rejection of trials. Hand and eye movements were recorded on-line by a PC during sessions and evaluated by custom software. In order to determine latency and duration of hand and eye movements, an off-line program for evaluation of movement trajectory parameters searched the movement record for the transgression and subgression of a vectorial velocity threshold of $10 \mathrm{~mm} / \mathrm{s}$ (which is equivalent to about $1 \%$ ). The beginning and the end of the movement were calculated as linear regressions in a $200 \mathrm{~ms}$ time window around these threshold points. Trials were rejected if (1) subjects made a saccade of more than $1^{\circ}$ of amplitude, (2) the hand movement started earlier than 100 or later than $500 \mathrm{~ms}$ after the go-signal, (3) movement duration was shorter than 100 or longer than $600 \mathrm{~ms}$, (4) the wrong hand was used for grasping, or (5) the index finger missed the target location. In total, 9.1\% of the trials had to be rejected.

For the statistical analysis the data were pooled for left and right hand movements, and we computed a two-way repeated measures ANOVA using the 
factors "DT position congruency" (congruent: discrimination target appearing at a to-be-grasped location vs. incongruent: discrimination target appearing at a not-to-be-grasped location) and "finger" (discrimination target appearing at a location in the upper hemifield, i.e., one that could be grasped by the index finger vs. discrimination target appearing at a location in the lower hemifield, i.e., one that could be grasped by the thumb). The factor "finger" was introduced to analyse a possible asymmetry in the selection of index finger- and thumb-grasped locations. In the remainder of this text, we will refer to DT locations in the upper hemifield as "index finger locations", to those in the lower hemifield as "thumb locations".

Procedure. There were two blocks of the dual-task paradigm with 128 trials each. Left and right hand were indicated in 64 trials, each with the DT being displayed 16 times at each of the four branch ends using " $E$ " and " $\exists$ ", randomly as discrimination item. In addition, subjects had to perform two control blocks, one of the "discrimination only, no grasping" task and another of the "grasping only, no discrimination" task. Two sessions were held with each subject. One session consisted of two blocks of the dual-task paradigm, preceeded by an additional practice-block. In the other session one block of each control condition was performed. The order of sessions was chosen randomly but balanced between subjects.

During the practice block subjects practiced fast and precise grasping using the correct hand indicated by the cue. In order to avoid learning effects with respect to shape and presentation of targets and distractors, mask items were displayed but did not change into targets and distractors during practice. For the same reason, the " $\mathrm{X}$ "'-shaped object to be grasped was replaced by a simple disc of the same diameter whose outline was also displayed on the screen instead of the object's outline.

\section{Results}

Movement performance. All subjects were able to respond correctly to the acoustic cue and executed prehension movements with satisfactory speed and precision after the initial practice block. As a typical example, Figure 4(a) shows the movement onset latency distribution for one subject. Like the one shown, distributions were monomodal for all subjects. In Figure 4(b), the index finger landing positions are depicted for the same subject. Note that the distance between the object's branch ends and the dots marking the landing positions is due to the fact that the position sensor was attached to the top of the index finger tip resulting in a small gap between the object's surface and the sensor.

Average movement onset latency was $331 \mathrm{~ms}$ (326 ms in the "graspingonly" control task). ANOVA showed that there was no significant main effect of DT position congruency, $F(1,5)=3.66, p=.11$, finger, $F(1,5)=4.63, p=$ 


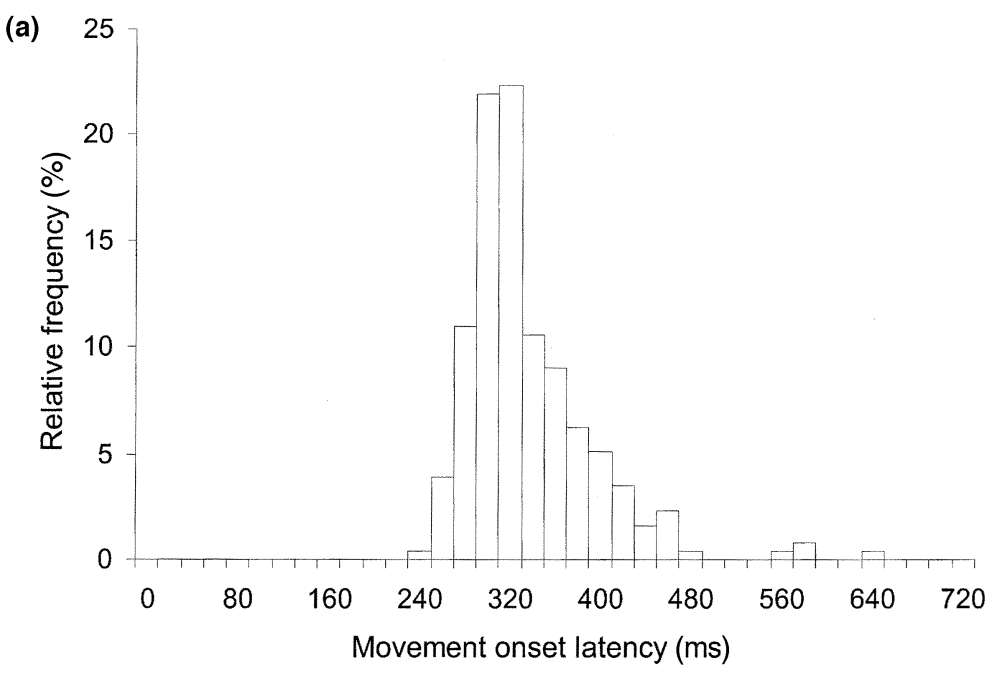

(b)

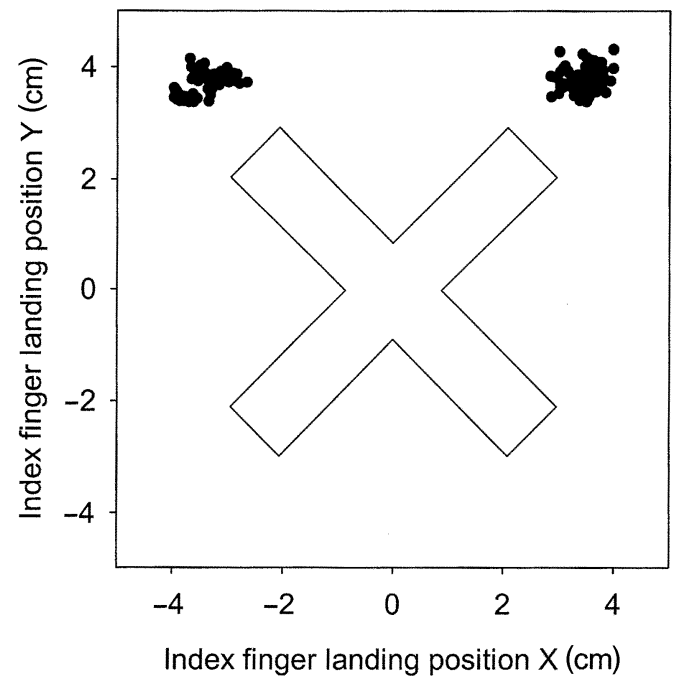

Figure 4. (a) Movement onset latency distribution for one subject. (b) Index finger landing positions for the same subject. The gap between landing positions and the object's branch ends is due to the position sensors being attached to the upper side of the fingers. 
.08 , and no interaction, $F(1,5)=0.01, p=.92$. Average movement duration was $271 \mathrm{msec}$ ( $272 \mathrm{msec}$ for the "'grasping-only"' control task). Like with movement onset latencies, there was no significant main effect of DT position congruency, $F(1,5)=2.62, p=.17$, and finger, $F(1,5)=0.29, p=.61$, and no interaction, $F(1,5)=0.03, p=.87$. As mentioned above, the fact that there was no effect of DT position congruency is important for the interpretation of the results of this experiment. It shows, that discrimination and prehension task did not interfere such that subjects would for example have detected the DT position during movement preparation, tried to identify " $\mathrm{E}$ " or " $\exists$ ', and only after this continued - possibly with a delay_processing the prehension task.

Perceptual performance. Discrimination performance is plotted in Figure 5. Average percentage correct discrimination was $68 \%$ for congruent versus $60 \%$ for incongruent discrimination target positions. ANOVA showed a significant main effect of DT position congruency, $F(1,5)=38.27, p<.01$, no significant effect of finger, $F(1,5)=0.01, p=.92$, and no interaction, $F(1,5)=1.11, p=$ .34 .

Summarizing the results of Experiment 1, data show that, during preparation of prehension movements, allocated attention determined by measuring percent correct discrimination is significantly higher for locations to-be-grasped than for locations not-to-be-grasped of the same object.

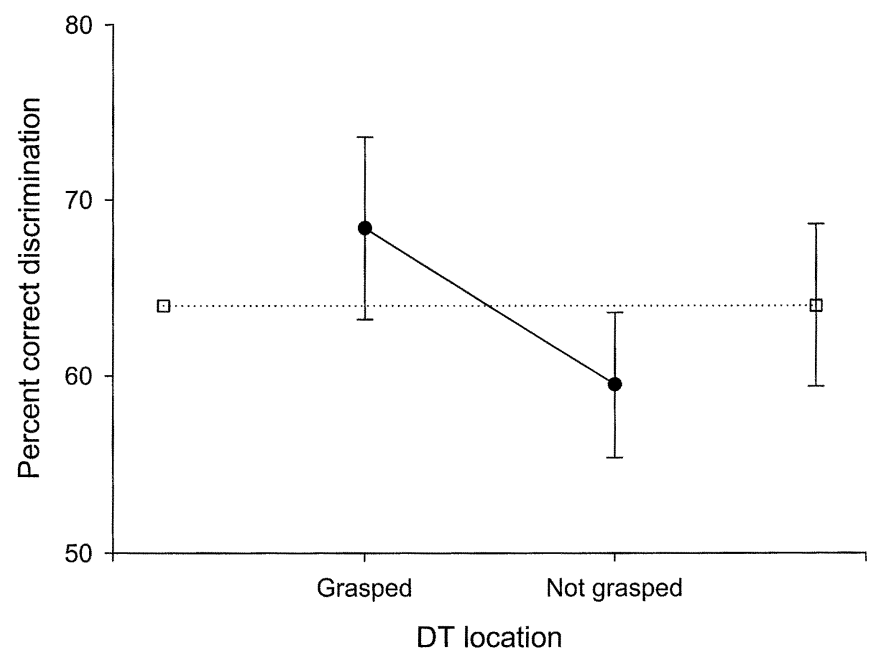

Figure 5. Experiment 1. Discrimination performance for discrimination target (DT) appearing at grasped and not grasped locations. Data for index finger and thumb locations are pooled. The dotted line indicates discrimination performance from the "no grasping - discrimination only" control task. Error bars denote standard errors. 


\section{EXPERIMENT 2}

The results of Experiment 1 show that, when not knowing the DT position, subjects' discrimination performance was significantly better when the target appeared at a to-be-grasped location than at a not-to-be-grasped location. One could however argue, that it was only convenient for the system to direct visual attention to the location to be grasped, because there was no cue where the DT would appear. In Experiment 2, we examined whether there is an obligatory coupling between selection for action and selection for perception. Therefore, DT position was kept constant during each block of trials and known to subjects. Provided that there is an obligatory coupling, one would assume that even if DT position is known in advance, discrimination performance will be better when the DT is at a to-be-grasped location than at a not-to-be-grasped location, because in the latter case programming the movement requires directing attention away from the DT (to the finger landing position). The second aim of Experiment 2 was to determine the time course of attentional selection, mainly to further examine whether index finger- and thumb-grasped locations are processed inhomogenously over time, such that for example, the thumb-grasped location would be selected first, and only later attention would be directed to the index finger grasped location. Therefore, we varied DT presentation onset relative to the movement go-signal.

\section{Methods}

Subjects. Eight right-handed subjects, others than those who had performed Experiment 1, participated in Experiment 2. They were aged 21-26 years, had normal vision, and had participated in reaching experiments before. However, they had not performed any grasping tasks and were naïve with respect to the aim of this study.

Experimental set-up, spatial layout of display, and data analysis. The experimental set-up, spatial layout of display, and criteria for rejection of trials were exactly like those described for Experiment 1 . In total, $8.3 \%$ of trials had to be rejected.

For the statistical analysis, the data were pooled for left and right hand movements and we computed a three-way repeated measures ANOVA using the factors "DT position congruency" (congruent vs. incongruent), "finger" (thumb vs. index finger), and "SOA" (100 ms, $300 \mathrm{~ms}, 500 \mathrm{~ms}$ ).

Discrimination and prehension task. Discrimination and prehension task were identical to those used in Experiment 1.

Temporal sequence of stimuli. The temporal sequence of stimuli was the same as in Experiment 1, except for the SOA that was now variable and could be 
100,300 , and $500 \mathrm{~ms}$. In addition, the DT appeared at a constant position during each block of trials. Subjects were informed about DT position before performing each block. To compensate the expected boost in percentage correct discrimination caused by subjects knowing the DT position, stimulus presentation time was reduced to $100 \mathrm{~ms}$.

Procedure. In total each subject underwent eight blocks of the dual task paradigm. There was one block of 72 trials for each of the four possible DT positions (at the branch ends of the object). Each of these blocks had to be performed twice. In each block right and left hand were indicated in 36 trials each, consisting of 12 trials for the SOAs 100, 300, and $500 \mathrm{~ms}$ respectively. At DT locations " $E$ " or " $\exists$ " were displayed randomly but balanced between trials. In addition, there were four blocks of the control condition "discrimination only, no grasping", one for each possible target position, and four blocks of the control condition "'grasping only, no discrimination'. In total, subjects had to perform 16 regular (nonpractice) blocks of trials which were split up into three sessions. Eight blocks of the dual task paradigm, preceded by a practice block like the one described with Experiment 1, were performed in one session. In addition, there was another session for each of the control conditions. The order of sessions was balanced between subjects.

\section{Results}

Movement performance. After the initial training block, all subjects were able to grasp the object with satisfactory speed and accuracy. Since movement onset latency distributions and index finger landing position patterns were very similar to those of Experiment 1, these results are not depicted here. Overall movement onset latency was $336 \mathrm{~ms}$ (334 ms for the "grasping-only" control task), which is very similar to the results of Experiment 1. ANOVA yielded neither a significant effect of any of the factors nor any significant interaction. As for movement duration, the average was $296 \mathrm{~ms}$ (295 ms for the " graspingonly" control task). Like for movement onset latency, ANOVA showed no significant main effects and no interactions.

As with Experiment 1, finding no significant effect of DT position congruency again shows that there was no interference between discrimination and prehension task. Table 1 summarizes the results of the variance analysis for movement duration and movement onset latency.

Perceptual performance. Percent correct discrimination is depicted in Figure 6. ANOVA yielded a significant main effect of $\operatorname{SOA}, F(2,14)=18.71, p$ $<.001$, and DT position congruency, $F(1,7)=45.04, p<.001$. There was no effect of finger, $F(1,7)=2.74, p=.14$, and no two-way interactions (see Table 2 
TABLE 1

Statistical values for the analysis of movement onset latency and movement duration in Experiment 2

\begin{tabular}{lrrrr}
\hline & Movement onset latency & \multicolumn{2}{c}{ Movement duration } \\
\hline SOA & $F(2,14)=1.19$ & $p=.33$ & $F(2,14)=0.34$ & $p=.72$ \\
Finger & $F(1,7)=0.03$ & $p=.87$ & $F(1,7)=2.19$ & $p=.18$ \\
DT position congruency & $F(1,7)=0.16$ & $p=.70$ & $F(1,7)=0.50$ & $p=.50$ \\
SOA $\times$ Finger & $F(2,14)=0.48$ & $p=.63$ & $F(2,14)=0.95$ & $p=.41$ \\
SOA $\times$ DT position congruency & $F(2,14)=1.00$ & $p=.39$ & $F(2,14)=0.54$ & $p=.59$ \\
Finger $\times$ DT position congruency & $F(1,7)=4.20$ & $p=.08$ & $F(1,7)=1.00$ & $p=.35$ \\
SOA $\times$ Finger $\times$ DT position congruency & $F(2,14)=0.47$ & $p=.63$ & $F(2,14)=0.93$ & $p=.42$ \\
\hline
\end{tabular}

for details). However, we found a significant three-way interaction, $F(2,14)=$ $5.53, p=.02$.

Percent correct discrimination-averaged over thumb and index finger locations - was $69.8 \%$ (congruent $=$ DT location grasped) vs. $63 \%$ (incongruent $=$ DT location not grasped) at SOA $100 \mathrm{~ms}, 72.7 \%$ vs. $65.8 \%$ at SOA $300 \mathrm{~ms}$ and $75.8 \%$ vs. $74.7 \%$ at SOA $500 \mathrm{~ms}$. Paired sample $t$-tests showed that percent correct discrimination was significantly better for grasped locations than for not grasped locations for SOA $100 \mathrm{~ms}(p<.001)$ and SOA $300 \mathrm{~ms}(p<.01)$ but not for SOA $500 \mathrm{~ms}(p=.76)$. With respect to SOA, $t$-tests with Bonferroni corrections showed that there was a significant difference in overall percentage correct discrimination between SOA $100 \mathrm{~ms}$ and SOA $500 \mathrm{~ms}(p<.01)$, between SOA $300 \mathrm{~ms}$ and SOA $500 \mathrm{~ms}(p=.03)$, and between SOA $100 \mathrm{~ms}$ and SOA 300 ms $(p=.04)$.

To further analyse the three-way interaction between SOA, DT position congruency, and finger, we computed simple main effects for the different values of SOA: There was a significant interaction between DT position congruency and finger at SOA $100 \mathrm{~ms}, F(1,7)=7.97, p=.03$. In contrast to that, at SOA $300 \mathrm{~ms}, F(1,7)=1.30, p=.29$, and SOA $500 \mathrm{~ms} F(1,7)=3.03, p=.13$, interactions were not significant. Pairwise comparisons showed that at SOA 100 msec discrimination performance was significantly better for congruent discrimination target position at thumb locations $(71 \%$ vs. $59.5 \% ; p<.001)$, while we found no significant difference for index finger locations $(68.6 \%$ vs. $66.5 \%$; $p=.38$ ). At SOA $300 \mathrm{~ms}$ this asymmetry between index finger and thumb locations seemed to reverse, yielding a bigger advantage of to-be-grasped over not-to-be-grasped locations for index finger (79.5\% vs. 68.6\%) than for thumb locations $(65.9 \%$ vs. $62.7 \%)$. However, this was-due to a high variability between subjects - not statistically significant. Data for percent correct discrimination are summarized in Table 3. 

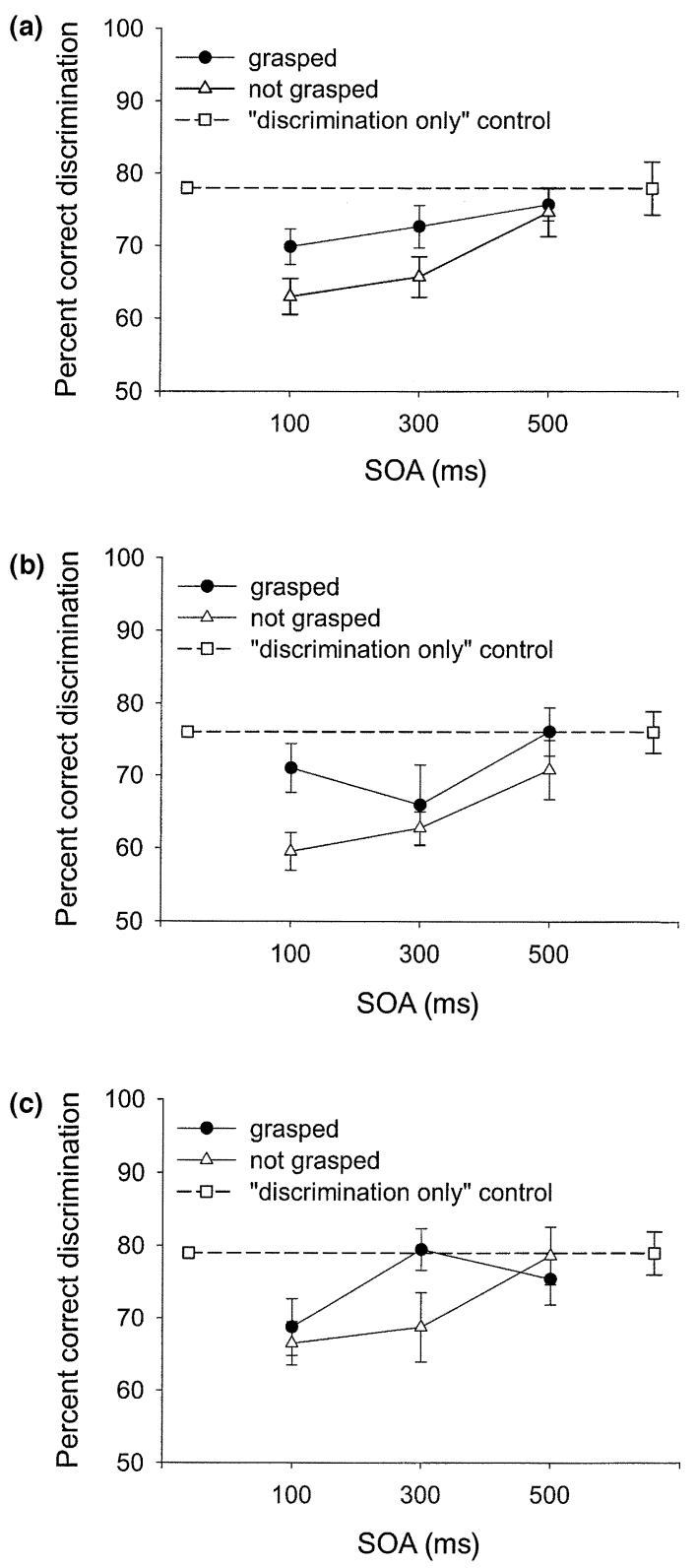

Figure 6. Experiment 2. Discrimination performance for discrimination target (DT) appearing at grasped and not grasped locations at different SOAs (relative to the movement go-signal) for (a) average over index finger and thumb locations, (b) thumb locations, and (c) index finger locations. The dotted lines indicate discrimination performance from the "no grasping — discrimination only" control task. Error bars denote standard errors.

\section{4}


TABLE 2

Statistical values for the analysis of percent correct discrimination in Experiment 2

\begin{tabular}{lrl}
\hline SOA & $F(2,14)=18.71$ & $p<.001$ \\
Finger & $F(1,7)=2.74$ & $p=.14$ \\
DT position congruency & $F(1,7)=45.04$ & $p<.001$ \\
SOA $\times$ Finger & $F(2,14)=1.24$ & $p=.32$ \\
SOA $\times$ DT position congruency & $F(2,14)=1.65$ & $p=.23$ \\
Finger $\times$ DT position congruency & $F(1,7)=0.75$ & $p=.42$ \\
SOA $\times$ Finger $\times$ DT position congruency & $F(2,14)=5.53$ & $p=.02$ \\
\hline
\end{tabular}

The results of this experiment show that, even when subjects knew the DT position, it was not possible to keep their visual attention fully on the DT when it was located at a part of the object that would not be grasped during preparation and early stages of the movement (SOAs $100 \mathrm{~ms}$ and $300 \mathrm{~ms}$ ). Otherwise, there would not have been any difference in percent correct discrimination between cases where the DT was at a to-be-grasped position and cases where it was not. However, for the very late stages of the movement (SOA $500 \mathrm{~ms}$ ) the difference was no longer significant. We speculate that programming motoric parameters, necessary for the execution of the movement, had been finished by that time and therefore, there was no more need to dorsally select to-be-grasped locations, which would have entailed a ventral selection of these very locations.

In addition to that, looking at the data for thumb and index finger locations separately, analyis of the three-way interaction revealed that during early stages of movement preparation (SOA $100 \mathrm{~ms}$ ) the advantage in percentage correct discrimination of to-be-grasped over not-to-be-grasped locations is only caused by thumb locations. This seems to be at variance with the results of Experiment 1 , where we found no significant difference between thumb and index finger locations. However, in Experiment 1, target presentation times were longer

TABLE 3

Percent correct discrimination for congruent (DT appeared at grasped branch end) and incongruent (DT appeared at a not grasped branch end) discrimination target position in Experiment 2

\begin{tabular}{|c|c|c|c|c|c|c|}
\hline \multirow[b]{2}{*}{$S O A$} & \multicolumn{2}{|c|}{ Index finger location } & \multicolumn{2}{|c|}{ Thumb location } & \multicolumn{2}{|c|}{ Average } \\
\hline & Congruent & Incongruent & Congruent & Incongruent & Congruent & Incongruent \\
\hline $100 \mathrm{~ms}$ & $68.6 \%$ & $66.5 \%$ & $71.0 \%$ & $59.5 \%$ & $69.8 \%$ & $63.0 \%$ \\
\hline $300 \mathrm{~ms}$ & $79.5 \%$ & $68.6 \%$ & $65.9 \%$ & $62.7 \%$ & $72.7 \%$ & $65.8 \%$ \\
\hline $500 \mathrm{~ms}$ & $75.4 \%$ & $78.6 \%$ & $76.1 \%$ & $70.8 \%$ & $75.8 \%$ & $74.7 \%$ \\
\hline
\end{tabular}


(140 ms in Exp. 1 vs. $100 \mathrm{~ms}$ in Exp. 2), and stimuli were presented at an SOA of $150 \mathrm{~ms}$. This means, that the target presentation interval stretched out to very late stages of movement preparation in Experiment 1. Therefore, over many trials, one might have measured a sort of average of attention being allocated for index finger and attention being allocated for thumb locations.

\section{DISCUSSION}

The main purpose of this study was to extend results from reaching tasks, suggesting coupling between (dorsal) selection for action and (ventral) selection for perception (Deubel et al., 1996, 1998), to prehension tasks. In contrast to these reaching tasks, where one small unstructured (with respect to motor parameter computation) object served as movement target, our prehension task involved a more complex computation of motoric parameters prior to the movement: First of all, there were two relevant "movement targets" for every single prehension movement, because thumb and index finger had to be directed to their final target locations on the object. Second, with respect to processing the to-be-grasped physical object itself, prehension tasks should cause some parts of the object (the to-be-grasped branch ends) to be more relevant to programming the motoric parameters than other parts (the not-to-be-grasped branch ends). Therefore, this study addressed two basic questions: First, whether the coupling between selection for action and selection for perception observed for simple reaching tasks could also be found for (with respect to programming motoric parameters) more complex prehension tasks and second, how a physical object would be processed when only parts of it were relevant for programming the motoric task.

Answering the first question, data from the two experiments presented in this study show that there is indeed a coupling between dorsal and ventral processing for both of the relevant parts (index finger's and thumb's landing position). Percentage correct discrimination, which served as a measure for visual attention, was always best when the discrimination target (DT) appeared -during movement preparation- at a location that would be grasped. In more detail, Experiment 1 was designed to observe coupling without the constraint of telling the subject in advance where the DT would appear. Results show that, during movement preparation, percentage correct discrimination was significantly better when the DT appeared next to a branch end of the object that would be grasped. Experiment 2 had two purposes: On the one hand, we wanted to find out whether coupling is obligatory or not, which could not be concluded from results of Experiment 1 . On the other hand, we were interested in the time course of coupling. Results from Experiment 2 show that even when subjects knew the position of the DT in advance (which was the case for this experiment because DT position was known and constant during a block of trials), percent correct discrimination for a given DT position was significantly better when the nearby branch end of the object 
would be grasped than for cases where it would not be grasped. This can be explained as follows: In cases where DT position was nearby a not-to-be-grasped branch end, "attention for action" (and, by assuming coupling, also "attention for perception") had to be withdrawn in order to attend to to-be-grasped locations. In contrast to that, when the DT position was nearby a to-be-grasped branch end, attention was focused on the very branch end where also the DT was located. As for the time course, data from Experiment 2 shows that coupling is obligatory before (SOA $100 \mathrm{~ms}$ ) and during the early stages of the movement (SOA $300 \mathrm{~ms}$ ). However, coupling vanishes after and possibly during the very late stages of the movement (SOA $500 \mathrm{~ms}$ ), for which no more significant difference in percentage correct discrimination between the two cases "DT nearby a to-be-grasped branch-end" and "DT nearby a not-to-be-grasped branch-end" was found. An interesting finding is that total percentage correct discrimination rises from early stages of movement preparation towards the end of the movement. This is possibly a direct consequence of the dual task paradigm. While during preparation of the movement resources are required for dorsal and ventral processing, close to the end of the movement or upon and after its completion dorsal processing should have been completed, and therefore, resources are freed which can now fully be used for processing the discrimination task.

Another interesting result is the fact that, during early stages of movement preparation, only for thumb locations there is a significant difference in percentage correct discrimination, facilitating the to-be-grasped location over the not-to-be grasped location. This may, in terms of attentional selection, reflect results of Wing and Fraser (1983) — see also Wing, Turton, and Fraser (1986) that suggest that the thumb is more important for the transport component of hand movements than the index finger. In this study, distance of thumb and index finger from an axis joining the wrist to the to-be-grasped object during reach-to-grasp movements was measured. Results showed that closing the hand for grasping is primarily due to index finger movement, whereas the thumb's position is rather invariant. The authors suggest that this might be due to the thumb serving as a stable reference point on the grasp surface used for guiding the transport of the hand to the object. Bearing in mind that the maximum grasp aperture is only reached after $80 \%$ of hand movement duration (see for example Jeannerod, 1981), it seems likely that computation of parameters for the transport component is prioritized before movement execution, while the manipulation component becomes fully relevant only later. One may speculate that therefore - during movement preparation - the landing position of the thumb is attended to, first. Previc (1990) has argued that for perceptual processes involved in visuomotor coordination in peripersonal space there is a bias on the lower visual field. One may therefore argue, that the attentional facilitation of the thumb positions we found in Experiment 2 during early stages of movement preparation (SOA $100 \mathrm{~ms}$ ) is due to this bias. We cannot completely rule out this possibility. However, we think that the data for SOA $300 \mathrm{~ms}$, where percentage 
correct discrimination is far better for index finger locations (i.e., the upper visual field) than for thumb locations show at least qualitatively that a lower visual field bias was not the dominating effect in our experiment.

The second question we asked above is concerned with attentional processing of a physical object when only parts of it are relevant to preparation of a motoric task. An interesting fact by itself is its obvious "attentional decomposition". The object (represented by its outline) is decomposed into movement relevant and movement-irrelevant parts. It has already been shown that there is a selection of one relevant object out of other irrelevant objects during preparation of goal-directed pointing movements (e.g., Deubel et al., 1998). The results presented in this study show that a similar selection process is at work when there is only one object, also selecting relevant over irrelevant "parts" of the visual field, which in this case belong to the same object. At this point, the question arises whether one can say anything about the nature of the selection process from our data. Was it specific to a spatial region, directing the focus of attention to the regions which would be the targets for thumb and index finger (spotlight or zoom lens model of spatial attention), or was it indeed object specific as, for example, the Visual Attention Model (VAM) of Schneider (1995) would propose?

There are numerous studies that have examined the "units" of attentional selection. While earlier results (e.g., Downing \& Pinker, 1985; Posner et al., 1980) supported the then dominant spotlight model, there has been growing evidence that indeed objects can be the units of attention (e.g., Baylis \& Driver, 1993; Duncan, 1984; Egly, Driver, \& Rafal, 1994). In addition, more recent results suggest, that spatial attention can be split among noncontiguous locations (Hahn \& Kramer, 1998; Kramer \& Hahn, 1995). In our paradigm, DTs were very close to the parts of the object relevant for the prehension task (namely its four branch ends) but not identical with them. Therefore, at first glance, one would favour spatial selection to be the underlying mechanism: The centre of attention is at one of the branch ends to be grasped, with attention dropping gradually going away from its centre but leaving nearby locations (containing the DT) also in an area of "enhanced" attentional levels. Obviously, the spotlight is not as wide as to include all target locations at a time. Otherwise, one would not have found differences in percentage correct discrimination in Experiment 1 between DTs near to-be-grasped and not-to-be grasped parts of the object, which all appeared at the same visual angle. Looking at the results from Experiment 2, in terms of the spotlight metaphor, a spotlight focused on a single location seems more plausible than a split spotlight. Data suggest that initially (SOA $100 \mathrm{~ms}$ ) attention is focused only on the thumb-grasped location. However, one cannot completely rule out the possibility of a split spotlight with different levels of attention at each of the to-be-grasped locations.

Moreover, we think that our results can also very well be explained by assuming object specific selection as the underlying mechanism. Using 
Schneider's (1995) VAM, which states that only one low-level-object at a time can be selected, one would need to assume that the physical object is decomposed into "subobjects", possibly the four branches of the cross. In addition, the to-be-selected subobjects would also have to include the adjacent DTs. During movement preparation, one of these subobjects - consisting of a branch of the cross and the adjacent DT-would be selected at a time. Since VAM does not make any statements about the actual composition of objects but only refers to so-called "internal" objects, which need not necessarily correspond to what one consciously perceives as an object, it can also perfectly account for our data. However, using our paradigm, one cannot decide, which mechanism of attentional selection-spatial or object-based selection-is at work.

In summary, our data provide on the one hand further evidence for an obligatory coupling of dorsal and ventral processing as proposed by models of Duncan (1996), Rizzolati et al. (1994) or Schneider (1995). On the other hand, they show that single objects are processed inhomogeneously during the preparation of prehension tasks in terms of attentional selection when some of their parts are more relevant to the task than others.

\section{REFERENCES}

Allport, D. A. (1987). Selection for action: Some behavioural and neurophysiological considerations of attention and action. In H. Heuer \& A. F. Sanders (Eds.), Perspectives on perception and action (pp. 395-419). Hillsdale, NJ: Lawrence Erlbaum Associates Inc.

Baylis, G., \& Driver, J. (1993). Visual attention and objects: Evidence for hierarchical coding of location. Journal of Experimental Psychology: Human Perception and Performance, 19, 451-470.

Bonfiglioli, C., \& Castiello, U. (1998). Dissociation of covert and overt spatial attention during prehension movements: Selective interference effects. Perception and Psychophysics, 60(8), $1426-1440$.

Bundesen, C. (1990). A theory of visual attention. Psychological Review, 97, 523-547.

Castiello, U. (1996) Grasping a fruit: Selection for action. Journal of Experimental Psychology: Human Perception and Performance, 22, 582-603.

Deubel, H., \& Schneider, W. X. (1996). Saccade target selection and object recognition: Evidence for a common attentional mechanism. Vision Research, 36, 1827-1837.

Deubel, H., Schneider, W. X., \& Paprotta, I. (1996). Visual attention and manual aiming: Evidence for obligatory and selective spatial coupling. Perception, 25, 13-14.

Deubel, H., Schneider W. X., \& Paprotta, I. (1998). Selective dorsal and ventral processing: Evidence for a common attentional mechanism in reaching and perception. Visual Cognition, 5, 81-90.

Downing, C., \& Pinker, S. (1985). The spatial structure of visual attention. In M. Posner \& O. S. M. Marin (Eds.), Attention and performance XI: Mechanisms of attention (pp. 171-187) Hillsdale, NJ: Lawrence Erlbaum Associates Inc.

Duncan, J. (1984). Selective attention and the organization of visual information. Journal of Experimental Psychology: General, 113, 501-517.

Duncan, J. (1996). Coordinated brain systems in selective perception and action. In T. Inui \& J. L. McClelland (Eds.), Attention and performance XVI. Information integration in perception and communication (pp. 549-578). Cambridge, MA: MIT Press. 
Duncan, J., \& Humphreys, G. W. (1989). Visual search and stimulus similarity. Psychological Review, 96(3), 433-458.

Egly, R., Driver, J., \& Rafal, R. (1994). Shifting visual attention between objects and locations: evidence for normal and parietal lesion subjects. Journal of Experimental Psychology: General, $123,161-177$.

Goodale, M. A., \& Milner, A. D. (1992). Separate visual pathways for perception and action. Trends in Neurosciences, 15, 20-25.

Hahn, S., \& Kramer, A. F. (1998). Further evidence for the division of attention among noncontiguous locations. In W. X. Schneider \& S. Maasen (Eds.), Mechanisms of visual attention: A cognitive neuroscience perspective (pp. 217-256). Hove, UK: Psychology Press.

Hoffman, J. E., \& Subramaniam, B. (1995). The role of visual attention in saccadic eye movements. Perception and Psychophysics, 57, 787-795.

Jackson, S. R., Jackson, G. M., \& Rosicky, J. (1995). Are non-relevant objects represented in working memory? The effect of non-target objects on reach and grasp kinematics. Experimental Brain Research, 102, 519-530.

Jeannerod, M. (1981). Intersegmental coordination during reaching at natural visual objects. In J. Long \& A. Baddeley (Eds.), Attention and performance IX (pp. 153-169). Hillsdale, NJ: Lawrence Erlbaum Associates Inc.

Kowler, E., Anderson, E., Dosher, B., \& Blaser, E. (1995). The role of attention in the programming of saccades. Vision Research, 35, 1897-1916.

Kramer, A. F., \& Hahn, S. (1995). Splitting the beam: Distribution of attention over noncontiguous regions of the visual field. Psychological Science, 6, 381-386.

Kritikos, A., Bennett, K., Dunai, J., \& Castiello, U. (2000). Interference from distractors in reach-tograsp movements. Quarterly Journal of Experimental Psychology, 53A (1), 131-151.

LaBerge, D., \& Brown, V. (1989). Theory of attentional operations in shape identification. Psychological Review, 96, 101-124.

Neumann, O. (1987). Beyond capacity: A functional view of attention. In H. Heuer \& A. F. Sanders (Eds.), Perspectives on perception and action (pp. 361-394). Hillsdale, NJ: Lawrence Erlbaum Associates Inc.

Posner, M. I. (1980). Orienting of attention. Quarterly Journal of Experimental Psychology, 32(1), $3-25$.

Posner, M. I., Snyder, C. R. R., \& Davidson, B. J. (1980). Attention and the detection of signals. Journal of Experimental Psychology: General, 109, 160-174.

Previc, F. (1990). Functional specialization in the lower and upper visual fields in humans: Its ecological origins and neurophysiological implications. Behavioral Brain Sciences, 13, 519-575.

Rizzolati, G., Riggio, L., Dascola, I., \& Umiltà, C. (1987). Reorienting attention across the horizontal and vertical meridians: Evidence in favor of a premotor theory of attention. Neuropsychologia, $25,31-40$.

Rizzolati, G., Riggio, L., \& Sheliga, B. M. (1994). Space and selective attention. In C. Umiltà \& M. Moscovitch (Eds.), Attention and performance XV: Conscious and unconscious information processing (pp. 231-265). Cambridge, MA: MIT Press.

Schneider, W. X. (1995). VAM: A neuro-cognitive model for visual attention control of segmentation, object recognition and space-based motor action. Visual Cognition, 2, 331-375.

Schneider, W. X., \& Deubel, H. (2002). "Selection-for-perception"' and Selection-for-spatial-motoraction are coupled by visual attention: A review of recent findings and new evidence form stimulus-driven saccade control. In W. Prinz \& B. Hommel (Eds.), Attention and performance XIX: Common mechanisms in perception and action. Oxford, UK: Oxford University Press.

Tipper, S. P., Howard, L. A., \& Jackson, S. R. (1997). Selective reaching to grasp: Evidence for distractor interference effects. Visual Cognition, 4(1), 1-38.

Treisman, A., \& Gelade, G. (1980). A feature-integration theory of attention. Cognitive Psychology, 12(1), 97-136. 
Wing, A. M., \& Fraser, C. (1983). The contribution of the thumb to reaching movements. Quarterly Journal of Experimental Psychology, 35A, 297-309.

Wing, A. M., Turton, A., \& Fraser, C. (1986). Grasp size and accuracy of approach in reaching. Journal of Motor Behavior, 18(3), 245-260.

Wolfe, J. M. (1994). Guided Search 2.0: A revised model of visual search. Psychonomic Bulletin and Review, 1, 202-238.

Manuscript received December 2001 Revised manuscript received August 2002 
